\title{
openheart The double jeopardy of chronic obstructive pulmonary disease and myocardial infarction
}

\author{
Shashank S Sinha, Hitinder S Gurm
}

To cite: Sinha SS, Gurm HS. The double jeopardy of chronic obstructive pulmonary disease and myocardial infarction. Open Heart 2014;1:e000010. doi:10.1136/openhrt-2013000010

Received 15 December 2013 Accepted 18 December 2013

Division of Cardiovascular Medicine, Department of Internal Medicine, University of Michigan, Ann Arbor, Michigan, USA

Correspondence to Dr Hitinder S Gurm; hgurm@med.umich.edu
Despite several therapeutic advances in the last few decades, chronic obstructive pulmonary disease (COPD) is a burgeoning cause of morbidity and mortality worldwide and is the fourth leading cause of death globally. ${ }^{1}$ Yet a curious paradox persists. As the only common disease for which the prevalence and mortality rates continue to rise, COPD still remains as a remarkably underdiagnosed and undertreated disease. ${ }^{1}$ The worldwide prevalence may be underestimated for a host of reasons including missed diagnosis, delays in establishing the diagnosis, variable definitions of COPD and the lack of age-adjusted estimates. COPD is of more than academic interest to cardiologists. Patients with COPD are at an increased risk of an array of acute cardiovascular events, including myocardial infarction (MI) and have increased short-term and long-term mortality compared with their non-COPD counterparts. $^{2}$ In fact, up to one-third of deaths in patients with COPD may be ascribed to cardiovascular disease. ${ }^{2}$ Cardiovascular mortality increases by $28 \%$ for every $10 \%$ decrement in forced expiratory volume in $1 \mathrm{s.}^{2}$ Furthermore, it has been shown that patients with COPD are undertreated with standard, guideline-based post-MI pharmacotherapy. Finally, the presence of COPD is associated with worse longterm outcome in patients undergoing percutaneous coronary intervention (PCI) or coronary artery bypass graft. ${ }^{3} 4$

COPD and coronary artery disease share common risk factor profiles and potentially mechanistic pathways. Tobacco use and old age contribute to elevated risk for both of these disease entities. In a recent study analysing 25857 patients with COPD in the Health Improvement Network database over a 2-year period, a COPD exacerbation was associated with over a twofold increased risk of MI during a 5-day period following the exacerbation. ${ }^{5}$ Notably, the risk returned to baseline within 5 days of treatment for the exacerbation. ${ }^{5}$ Although the exact pathogenesis remains to be elucidated, numerous plausible mechanisms have been postulated. One hypothesis is that an acute chronic airway inflammation resulting from COPD exacerbations triggered by either viral or bacterial airway infection may lead to systemic inflammation, as manifested by elevations in $\mathrm{C}$ reactive protein and leucocyte counts, both markers of increased cardiovascular morbidity. ${ }^{5}$ Inflammation precipitates cytokine release, which combined with a hyper-adrenergic state, promotes macrophage uptake of low-density lipoprotein particles and augments adhesion of leucocytes to arterial endothelium, thereby increasing the risk for MI. ${ }^{5}$

The impact of COPD on outcome of patients with MI has been only recently investigated.

The study by Andell $e t$ at published in this issue of Open Heart provides a compelling characterisation examining the prognostic impact of COPD in patients with acute MI. In a large Swedish cohort of 81191 patients with MI, patients with COPD frequently manifested an atypical presentation, endorsing dyspnoea more frequently and chest pain less frequently compared with patients without COPD. Importantly, patients with COPD were less likely to present with an ST-segment elevation MI and less often underwent PCI. It is noteworthy that patients with COPD less often received guideline-based, secondary post-MI medications with proven mortality benefit, including aspirin, clopidogrel, $\beta$-blockers, ACE inhibitors and statins. Patients with COPD were significantly more likely to die compared with those without COPD over the follow-up period. Perhaps the most compelling finding of the study was that the unadjusted 1-year mortality for patients with COPD after an MI (HR $1.86,95 \%$ CI 1.76 to 1.98 ) was substantially reduced after adjusting for baseline characteristics and comorbidities (HR 1.32, 95\% CI 1.24 to 1.40) and diminished further after adjusting for different treatment patterns 
(HR 1.14, 95\% CI 1.07 to 1.21), yielding only a modest, yet statistically significant increase in adjusted mortality as compared with their non-COPD counterparts once treatment differences were adjusted for.

While observational studies cannot ascribe causality, this analysis raises the provocative possibility that more consistent application of guideline-recommended therapy may help partially negate the survival hazard associated with COPD in this population. Nonetheless, the study by Andell and colleagues must be interpreted with certain caveats. As the study employed clinical rather than spirometric definitions of COPD, it is possible that several patients may have been misclassified. For instance, several patients with asthma could have potentially been included in the COPD group. Similarly, several patients with COPD could have been misclassified as non-COPD as the 6\% prevalence reflected in the SWEDEHEART registry is lower than the estimated $9-10 \%$ prevalence of COPD in Sweden. Previous studies have demonstrated an association between COPD severity and clinical outcome. ${ }^{7}$ The absence of pulmonary function data in the current study meant that the authors were unable to explore this relationship. Nonetheless, the study strongly suggests that patients with COPD have a substantially worse prognosis with respect to mortality after MI and adds to the growing body of data supporting the increased hazard imparted by the co-occurrence of coronary artery disease and COPD.

What can be done to negate the increased hazard of cardiovascular mortality in patients with COPD (figure 1)? The dramatic reductions in cardiovascular mortality in the general population have been predominantly driven by

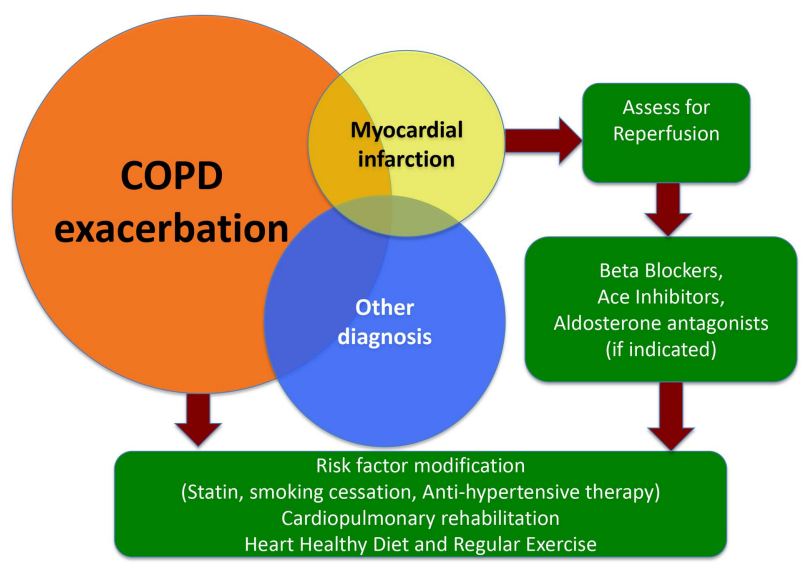

Figure 1 The recommended approach to patients with chronic obstructive pulmonary disease (COPD) presenting with dyspnoea. An atypical presentation of myocardial infarction (MI) should be considered in every patient presenting with COPD exacerbation with the understanding that MI may coexist with another acute illness in these patients. Patients with COPD and MI should be urgently assessed for revascularisation via percutaneous coronary intervention and started on guideline-based therapy. Any hospitalisation in a patient with COPD, even in the absence of an $\mathrm{MI}$, should be considered an opportunity to assess and optimise the coronary risk factors. better risk factor control and optimisation of primary and secondary medical therapy, and the same approach needs to be applied to patients with COPD. ${ }^{9}$ Although $\beta$-blockers have been shown to reduce mortality and the risk of reinfarction after MI, they are vastly underutilised in patients with COPD, perhaps, due to historical concerns regarding bronchospasm. Cardioselective $\beta$-blockers are less likely to induce bronchospasm and can be titrated starting at the lowest available dose (table 1). In our practice, we favour the use of bisoprolol, which can be initiated at $1.25 \mathrm{mg}$ daily, then titrated to $2.5 \mathrm{mg}$ daily after $1-2$ weeks, and increased to $5 \mathrm{mg}$ daily after 4 weeks. In the recently published population-based cohort study of 1063 patients with COPD in the UK Myocardial Ischaemia National Audit Project (MINAP), treatment with $\beta$-blockers started during the hospital admission for MI was associated with a significant mortality benefit over a median follow-up of 2.9 years (fully adjusted HR $0.50,95 \%$ CI 0.36 to 0.69 ). ${ }^{2}$ Patients already taking a $\beta$-blocker before their MI also had a significant survival benefit (HR 0.59; 95\% CI 0.44 to 0.79 ). ${ }^{2}$ However, only $38.6 \%$ of patients with COPD received a $\beta$-blocker during the hospital admission for MI. ${ }^{2}$

The findings published in this observational study by Andell $e t a l^{6}$ ideally need to be validated in a prospective, large, multicenter randomised controlled trial. However, it appears unlikely that such a trial will be performed in the near future and based on data extrapolated from patients with non-COPD, we must ensure that patients with COPD are discharged on the guideline-based regimens for standard post-MI care. This includes dual antiplatelet therapy, $\beta$-blockers as discussed above, ACE inhibitors and statins. A high index of suspicion must be employed in patients with COPD as they frequently present with dyspnoea and atypical symptoms and an MI can be easily missed. Even in the absence of MI, every hospitalisation for COPD should be an opportunity to optimise risk factor control to reduce the risk of future MI. Use of standardised check lists and discharge sheets can be highly effective in optimising uptake of preventive therapy in those with MI and those with COPD.

Finally, the role of cardiopulmonary rehabilitation merits consideration. Current guidelines recommend cardiopulmonary rehabilitation as an evidence-based, multidisciplinary and cost-effective intervention that leads to improved health in patients with COPD and MI. However, a recent national survey of hospital-based

\begin{tabular}{lcc} 
Table 1 & Cardioselectivity of $\beta$-blockers \\
\hline $\begin{array}{l}\text { Cardioselective } \\
\boldsymbol{\beta} \text {-blockers }\end{array}$ & $\begin{array}{l}\text { Relative } \boldsymbol{\beta 1 / \beta 2} \\
\text { selectivity }\end{array}$ & $\begin{array}{l}\text { Usual dose } \\
\text { range (mg/day) } \\
\text { (frequency per day) }\end{array}$ \\
\hline Celiprolol & 69 & $200-400(1)$ \\
Metoprolol & 74 & $50-200(1-2)$ \\
Betaxolol & 93 & $10-20(1)$ \\
Bisoprolol & 103 & $2.5-10(1)$ \\
Nebivolol & 321 & $5-40(1)$ \\
\hline
\end{tabular}


pulmonary rehabilitation in patients with COPD in Sweden demonstrated that only $0.2 \%$ of the country's estimated COPD population participated in a hospitalbased pulmonary rehabilitation programme. ${ }^{10}$ Yet pulmonary rehabilitation has been shown to improve exercise capacity, reduce dyspnoea and fatigue, improve healthcare quality of life and reduce hospitalisations with the greatest benefit derived in GOLD stages II-IV. ${ }^{11}$ Further investigations, including randomised controlled trials examining the clinical efficacy of cardiopulmonary rehabilitation in patients with COPD and MI, are warranted. This is especially pertinent since the mortality benefit of cardiac rehabilitation in patients with MI has recently been challenged in light of the negative findings of the Rehabilitation After Myocardial Infarction Trial (RAMIT). ${ }^{12}$ Nonetheless, it would be premature to abandon cardiopulmonary rehabilitation in this population and especially in those with COPD. Patients with COPD who suffer MIs represent a high-risk cohort that clearly merits closer surveillance and aggressive pharmacological and non-pharmacological intervention to alleviate the increasing burden of this global epidemic.

Competing interests HSG received research funding from the National Institute of Health and Agency for Healthcare Research and Quality.

Provenance and peer review Commissioned; internally peer reviewed.

Open Access This is an Open Access article distributed in accordance with the Creative Commons Attribution Non Commercial (CC BY-NC 3.0) license, which permits others to distribute, remix, adapt, build upon this work noncommercially, and license their derivative works on different terms, provided the original work is properly cited and the use is non-commercial. See: http:// creativecommons.org/licenses/by-nc/3.0/

\section{REFERENCES}

1. Yusen RD. Evolution of the GOLD documents for the diagnosis, management, and prevention of chronic obstructive pulmonary disease. Controversies and questions. Am J Respir Crit Care Med 2013;188:4-5.

2. Quint JK, Herrett E, Bhaskaran K, et al. Effect of beta blockers on mortality after myocardial infarction in adults with COPD: population based cohort study of UK electronic healthcare records. BMJ 2013;347:f6650.

3. Selvaraj CL, Gurm HS, Gupta R, et al. Chronic obstructive pulmonary disease as a predictor of mortality in patients undergoing percutaneous coronary intervention. Am J Cardiol 2005;96: 756-9.

4. Leavitt BJ, Ross CS, Spence B, et al. Long-term survival of patients with chronic obstructive pulmonary disease undergoing coronary artery bypass surgery. Circulation 2006;114(1 Suppl):1430-4.

5. Donaldson GC, Hurst JR, Smith CJ, et al. Increased risk of myocardial infarction and stroke following exacerbation of COPD. Chest 2010;137:1091-7.

6. Andell P, Koul S, Martinsson A, et al. Impact of chronic obstructive pulmonary disease on morbidity and mortality after myocardial infarction. Open Heart Published Online First: xx Jan 2014. doi:10.1136/openhrt-2013-000002

7. Curkendall SM, Lanes S, de Luise C, et al. Chronic obstructive pulmonary disease severity and cardiovascular outcomes. Eur $J$ Epidemiol 2006;21:803-13.

8. Curkendall SM, DeLuise C, Jones JK, et al. Cardiovascular disease in patients with chronic obstructive pulmonary disease, Saskatchewan Canada cardiovascular disease in COPD patients. Ann Epidemiol 2006;16:63-70.

9. Gurm HS, Eagle KA. Channelling regional registries for optimization of cardiac care: lessons from around the world. Eur Heart $J$ 2013;34:83-5.

10. Wadell K, Janaudis Ferreira T, Arne M, et al. Hospital-based pulmonary rehabilitation in patients with COPD in Sweden-a national survey. Respirat Med 2013;107:1195-200.

11. Garvey C, Spruit MA, Hill K, et al. International COPD Coalition Column: pulmonary rehabilitation-reaching out to our international community. J Thorac Dis 2013;5:343-8.

12. West RR, Jones DA, Henderson AH. Rehabilitation after myocardial infarction trial (RAMIT): multi-centre randomised controlled trial of comprehensive cardiac rehabilitation in patients following acute myocardial infarction. Heart 2012;98:637-44. 